\title{
Analyzing K-12 Blended MOOC Learning Behaviors
}

\author{
Robert Gold \\ Oberlin College \\ Oberlin, United States \\ robertgold@csail.mit.edu
}

\author{
Erik Hemberg \\ ALFA, MIT CSAIL \\ Cambridge, United States \\ hembergerik@csail.mit.edu
}

\author{
Una-May O'Reilly \\ ALFA, MIT CSAIL \\ Cambridge, United States \\ unamay@csail.mit.edu
}

\begin{abstract}
We investigate student learning behaviors in a Massive Open Online Course with in-person components. Our goal is to improve the design of the course through learning analytics. The programming language taught, App Inventor, is a drag-anddrop language to create Android applications. We visualize and quantify student behaviors such as automatic and manual saving of code, video sections viewed, and the various forms of knowledge required to understand the course material. It appears students are less likely to go from course material that teaches procedures to other material that teaches procedures than we would expect, and rarely review previous topics covered in the course. We also find students tend to save marginally less at the beginning and end of sessions. However, since the data set is small, our conclusions are limited.
\end{abstract}

\section{Author Keywords}

Blended MOOCs; App Inventor; learning analytics; learning design; resource usage; knowledge type.

\section{CCS Concepts}

-Applied computing $\rightarrow$ E-learning; •Social and professional topics $\rightarrow$ Computational thinking; K-12 education;

\section{INTRODUCTION}

It is difficult for instructors to monitor student progress or provide feedback in Massive Open Online Courses (MOOCs) to the extent possible in physical classrooms [8] [1]. Instructor feedback can be essential to introductory programming classes because programming is hands-on and difficult to learn and teach [1] [5]. Programming used to be considered a specialized skill set. Therefore, much of the knowledge regarding how to teach programming is targeted towards post-secondary education. Now, however, programming is considered more mainstream [4]. In the absence of level-specific knowledge, teaching programming to K-12 students in an online setting is particularly challenging. To address this issue, some MOOCs have in-person components. These are called blended MOOCs [3]. This structure allows for students to be largely independent yet still receive some instructor feedback and monitoring.

Permission to make digital or hard copies of all or part of this work for personal or classroom use is granted without fee provided that copies are not made or distributed for profit or commercial advantage and that copies bear this notice and the full citation on the first page. Copyrights for components of this work owned by others than the author(s) must be honored. Abstracting with credit is permitted. To copy otherwise, or republish, to post on servers or to redistribute to lists, requires prior specific permission and/or a fee. Request permissions from permissions@ acm.org.

L@S '20, August 12-14, 2020, Virtual Event, USA.

(C) 2020 Association for Computing Machinery.

ACM ISBN 978-1-4503-7951-9/20/08 . \$15.00

http://dx.doi.org/10.1145/3386527.3406743
However, the mixture of online and in-person content adds noise to the data as we are unsure what students were told in person (ex. "Skip unit 5").

We focus on a blended MOOC taught by the Hong Kong University of Science and Technology in $2018^{1}$. The in-person portion of the course consists of workshops for students. The course teaches K-12 students App Inventor, a drag and drop programming language to create Android Applications, and computational thinking. Computational thinking is the set of problem-solving methods used to express problems and their solutions in ways that computers can execute [7]. The App Inventor programming is done on a separate server.

Our research questions are: What patterns of learning do students exhibit in a blended MOOC environment? How do students progress throughout the course and within individual problems? What difficulties do students have with the online course material (resources)? What habits and skills should they develop to improve their studies in such an environment?

We answer these questions by quantifying and visualizing behaviors such as automatic and manual saving of code, video sections viewed, and student interactions with different types of resources, which we categorize by the "forms" of knowledge required to understand the material (knowledge types). From these data, we infer how students work and whether that matches the design of the course (learning design). By investigating resource usage patterns and knowledge types, we try to understand how students interact with different types of course material.

Students are not required to follow the course from start to finish, which we call the intended course trajectory. Yet, we find students do. We examine this adherence to the intended course trajectory in combination with resource knowledge types. Finally, reviewing course material has been shown to be an effective learning technique, so we look at whether students review materials [6].

\section{COURSE CONTEXT}

The course uses the Open edX platform, which we use to collect clickstream data. User code is extracted from the App Inventor Server. User code is automatically saved every five minutes if the code has changed from the previous save state. Students may also manually save at any time. As a result, we only see changes from one save state to another, rather than

\footnotetext{
${ }^{1}$ https://learn. familylearning.hk/courses/course-v1: HKUST+appinv2+2019_Q4_R1/about
} 
every change a student makes. We take this as evidence that the save data is noisy.

The data is derived from approximately 300 students, only about 100 of whom interact with both the edX course and the App Inventor Server. For questions pertaining to user code, we only use data from those 100 students with both server and course activity. Otherwise, we use the data from all 300 students. It appears the only difference between these cohorts is that students with both course and server activity tend to have more daily interaction with resources.

The data are separated into clickstream data (logs of how users interact with the course material) and code save states, which are tied to a user and time of occurrence. We define a user save state as the characteristics of a user's code at any given save point. Note, for clickstream data, we cannot know if students use these resources or if they are accidental clicks. We are also unable to say if the user is actively engaged with a resource or if they are idle.

Clickstream data allows us to track how students interact with each resource in the edX course. The edX data do not provide all the information necessary to see an accurate progression of students learning. So, we ran a web scraper to obtain the order that resources appear within the course, accurate resource names and all possible URLs for every resource.

Each resource is categorized based on a revised Bloom's taxonomy [2] by learning design experts according to what types of knowledge are required to understand the material. Factual knowledge is the basic information necessary to understand a topic (ex. vocabulary). Conceptual knowledge refers to the interrelationships among facts and how they function within larger structures. Procedural knowledge consists of methods, algorithms and skills used to solve problems, and the criteria for when it is appropriate to use them. A resource can have multiple knowledge types.

\section{METHODS}

\section{Aggregated Saves}

We aggregate code save states to visualize how often students save code throughout sessions. We define a session as a set of resource interactions surrounded by no interactions for at least $\delta$ amount of time. We examine code save states on the App Inventor Server across all sessions. By definition, a session on the App Inventor Server must end and start with a save. Therefore, we ignore the first and last save in every session. We run this process for all sessions with $\delta$ values between 1 and 40 minutes. We look at this time range because $80 \%$ of all saves are within 40 minutes of another save. Sessions longer than 40 minutes appear to be less representative of the data. For each run, we calculate the percentage of the number of times each student saves at every point in a session out of the total number of saves each student makes in that session. We then take the median percentage of saves at each point across all sessions and students.

\section{Resource Reviews}

We classify a review as a transition from a resource that appears later in the course to a resource that occurs earlier in the course. For example, a review could be a transition from resource 9 to resource 2 . We can say any pair of resources $\left(n_{i}, n_{j}\right)$ is a review if and only if $j<i$. We call $n_{i}$ the origin resource and $n_{j}$ the destination resource.

To track whether students review resources, we look at clickstream data excluding the App Inventor Server page and the homepage. We attempt to filter out accidental clicks and students quickly reviewing a resource but then realizing it is not relevant. We do this by only including reviews in which a student spends at least three minutes on the destination resource. We define the time spent on a resource as the time spent between subsequent clicks in a clickstream log.

\section{Knowledge Type Transitions}

We quantify the likelihood a student will transition from one resource knowledge type to another within a session, where the session threshold is two hours.

One transition can include multiple knowledge type transitions. For example, if the user moves from a resource which contains factual and procedural knowledge to a resource which contains conceptual and procedural knowledge, that would be considered a transition from factual to conceptual, factual to procedural, procedural to conceptual, and procedural to procedural.

Since the distribution of resource transition knowledge types is unequal, we look at the percentage of transitions from each knowledge type to all three knowledge types in comparison to the percentage of resources that contain each knowledge type.

Also, since knowledge types are often followed by the same knowledge types in the intended course trajectory, we consider the distribution of repeated knowledge type transitions in the context of the intended course trajectory.

\section{Video Fast Forwards and Rewinds}

We aggregate clickstream data by video within the course to see whether students are reviewing or skipping sections of videos. We look at the clickstream event seek_video which represents when a user fast forwards or rewinds a video.

A seek_video event contains two time fields: the time that the seek_video event occurred within the video and the time in the video moved to. We use this to determine whether a user has fast forwarded or rewound. We will refer to fast forwards as forward seeks and rewinds as backward seeks.

Prior to every seek_video event a play_video event occurs, which represents when the user plays the video. Every play_video includes the time within the video that the video starts from. Note, in about $6.8 \%$ of seek_video events, the time within the video that the event occurs is earlier than the time in the play_video event before it. These data are invalid because a play_video event always precedes a seek_video event. For this reason, we take this as evidence that the clickstream data is noisy.

\section{RESULTS}

\section{Aggregated Saves}

Based on the data shown in Figure 1, it appears students save slightly less towards the beginning and end of a session. To 


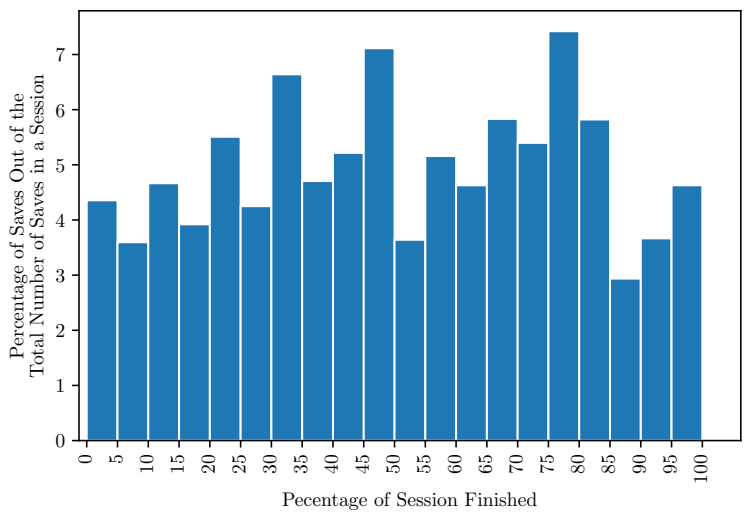

Figure 1. Distribution of Saves at Different Points in a Session: we determine the percentage of the number of times each student saves at every point within a session out of the total number of saves each student makes. We do this for sessions with $\delta$ values between 1 and 40 minutes. We then take the median percentage of saves at each point within the sessions across all students. The $x$-axis shows how far through a session a save occurs. The $y$-axis shows the percentage of total saves made at each point within the session. We see students tend to save slightly less at the beginning and end of sessions.

support this observation, we run Kruskal-Wallis tests on the beginning and middle, middle and end, and beginning and end sections of the aggregated distribution. We classify the beginning section to be saves that occur within the first $10 \%$ of sessions, the middle section to be saves that occur between $45 \%$ and $55 \%$ of the way through sessions, and the end section to be saves that occur in the last $10 \%$ of sessions. We also compare medians to estimate the effect size. According to Table 4, there is a small but statistically significant difference in the number of saves throughout a session. Therefore, it is possible students save slightly more in the middle of a session than at the beginning and end.

\section{Resource Reviews}

As shown in Table 1, there is little evidence to suggest students review resources. Many of the reviews that do occur make sense in context of the course. For example, "Component Property" contains knowledge directly related to "Label component" (highlighted in Table 1).

\section{Knowledge Type Transitions}

In Table 2, we see that transitions made by students (student transitions) from factual to factual knowledge types occur $34 \%$ of the time, while only $19 \%$ of all transitions contain factual knowledge, a ratio of about $\frac{5}{3}$. We see about the same ratio for conceptual to conceptual transitions. Yet, for procedural to procedural transitions, we see a ratio of about $\frac{14}{13}$. In addition, according to Table 3, procedural resources follow other procedural resources about $13 \%$ more than factual resources follow factual resources and about $20 \%$ more than conceptual resources follow conceptual resources. Therefore, we would expect a procedural to procedural transition to be as, if not more, likely to occur than a factual to factual or a conceptual to conceptual transition.

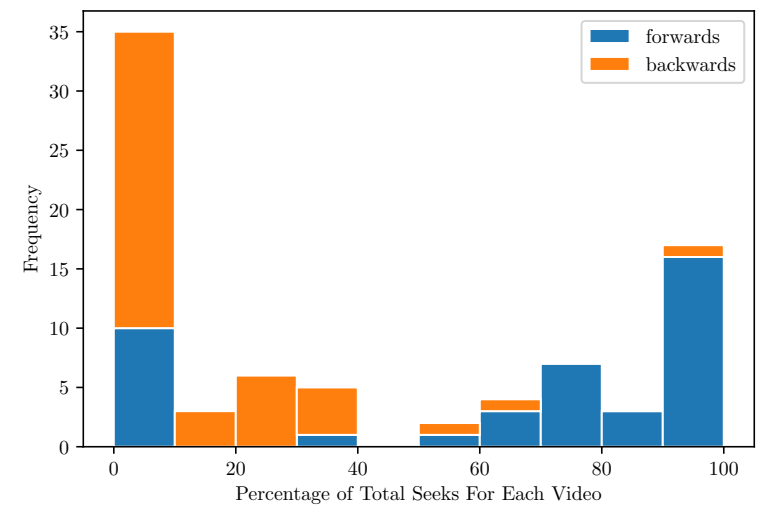

Figure 2. Video Fast Forwards and Rewinds: this figure shows the distribution of fast forwards (forwards seeks) and rewinds (backwards seeks) per video. The $x$-axis shows the percentage of each type of seek within a video and the $y$-axis shows the frequency at which that percentage occurs.

\section{Video Fast Forwards and Rewinds}

As indicated by Figure 2, backward seeks typically make up $40 \%$ or less of seeks within a video, with $0 \%$ being the mode. In contrast, most forward seeks make up $60 \%$ or more of the seeks within a video, with $100 \%$ being the mode. It appears that in comparison to forwards seeks, students rarely seek backwards. One possibility is that these forward seeks are representative of students skipping content. Another possibility is that students might watch a video once, then come back to it at a later time and fast forward to the relevant content.

\section{CONCLUSION AND FUTURE WORK}

In order to improve distance learning, we must understand students' learning patterns. Our investigation focused on patterns in how students solve computational problems. In essence, we attempted to infer students' computational thinking processes.

We find that students are less likely to go from a resource with procedural knowledge to one with procedural knowledge than we would expect, appear to not review resources often and fast forward videos more often than rewind. In addition, we see students tend to save slightly less at the beginning and end of sessions.

However, our data set is small, and thus our interpretations are limited. It would be best to run this analysis with data from multiple runs of the course. In addition, some of the clickstream data is corrupt such as some of the seek_video events. In fact, we do not know if any clicks in the clickstream were accidental, if the students attempted to learn from the resources, or if the users were idle after a click. Further, the App Inventor Server only collects data in five-minute intervals, which may or may not be often enough to accurately track how students save code. We also only know what students do, not what they are thinking. One solution to this could be to include a survey at the end of certain resources that asks questions such as "What did you find hard about this problem?". In addition, we do not know what the students were told during the inperson portions of the course, which adds noise to the data. We also arbitrarily defined the threshold for a transition to be 


\begin{tabular}{lrr} 
Origin Resource & Destination Resource & Count \\
\hline Waste Data And Statistics & Code2App Student Advanced Workshop Mar 2 & 2 \\
Using Emulator & Component Property & 2 \\
Label Component & Syllabus & 2 \\
App Inventor Project and Starting HelloWorld Project & Non-visible TextToSpeech Component & 3 \\
Homework 1 & Code2App Student Advanced Workshop Mar 2 & 3 \\
Background Information & Homework 1 & 3
\end{tabular}

Table 1. Resource Reviews: this shows what resources students review. The "Origin Resource" column shows the origin resource, the "Destination Resource" column shows the destination resource, and the "Count" column shows the amount of times each review occurred. It appears students do not review resources often. The highlighted row is an example of a review which makes sense in context of the course.

\begin{tabular}{lrrr} 
& \multicolumn{3}{c}{ Destination Type } \\
\cline { 2 - 4 } Origin Type & Factual & Conceptual & Procedural \\
\hline All & $19 \%$ & $16 \%$ & $65 \%$ \\
Factual & $34 \%$ & $13 \%$ & $52 \%$ \\
Conceptual & $16 \%$ & $26 \%$ & $58 \%$ \\
Procedural & $15 \%$ & $14 \%$ & $71 \%$
\end{tabular}

Table 2. Knowledge Type Transitions: The row named "All" shows the percentage of student transitions that contain a certain knowledge type. The other rows show the percentage of student transitions that go from an origin resource with a knowledge type to a destination resource with some knowledge type out of the total number of transitions that include the knowledge type of the origin resource.

\begin{tabular}{lrr} 
Factual & Conceptual & Procedural \\
\hline $62 \%$ & $70 \%$ & $83 \%$
\end{tabular}

Table 3. Knowledge Type Repeats: this shows the percentage of transitions in the intended course trajectory that go from one knowledge type to the same knowledge type out of all transitions containing that knowledge type.

\begin{tabular}{lrr} 
Comparison & Change in \% Saves & p-value \\
\hline Beginning vs. Middle & $-1 \%$ & $<0.001$ \\
Middle vs. End & $2 \%$ & $<0.001$ \\
Beginning vs. End & $1 \%$ & $<0.001$
\end{tabular}

Table 4. Kruskal Wallis Tests: we perform Kruskal-Wallis tests to compare the beginning, middle and end sections of the aggregated saves. The "Comparison" column shows the two sections being compared. The "Change in \% Saves" column shows the difference in medians between the two sections. The " $p$-value" column shows the p-value of the KruskalWallis test between the two sections.

considered a review to be three minutes. In future work, we will explore different review thresholds. Additionally, we only considered transitions in sessions with $\delta$ values of two hours when analyzing knowledge type transitions. In future work, we will analyze knowledge type transitions in sessions with different $\delta$ values. In addition, to better understand student habits, we will determine whether there are certain periods of time when students save code more frequently, relative to their time zones. Lastly, we will attempt to infer whether video fast forwards are representative of students skipping content or reviewing it by checking if they have viewed the video before.

\section{REFERENCES}

[1] Ayesha Bajwa, Ana Bell, Erik Hemberg, and Una-May Oreilly. 2019. Analyzing Student Code Trajectories in an Introductory Programming MOOC. 2019 IEEE Learning With MOOCS (LWMOOCS) (2019), 53-58. DOI :

http://dx.doi.org/10.1109/1wmoocs47620.2019.8939666

[2] W. A. L. Blyth, B. S. Bloom, and D. R. Krathwohl. 1966. Taxonomy of Educational Objectives. Handbook I: Cognitive Domain. British Journal of Educational Studies 14, 3 (1966), 119. DOI :

http://dx.doi.org/10.2307/3119730

[3] Curtis Jay. Bonk and Charles R Graham. 2007. The handbook of blended learning: global perspectives, local designs. Pfeiffer.

[4] Google Inc. \& Gallup Inc. 2016. Trends in the State of Computer Science in U.S. K-12 Schools. (2016). https://services.google.com/fh/files/misc/ trends-in-the-state-of-computer-science-report.pdf

[5] B. Kaučič and T. Asič. 2011. Improving introductory programming with Scratch?. In 2011 Proceedings of the 34th International Convention MIPRO. IEEE, 1095-1100.

https://ieeexplore.iee.org/document/5967218

[6] Yanbang Wang, Nancy Law, Erik Hemberg, and Una-May O'Reilly. 2019. Using Detailed Access Trajectories for Learning Behavior Analysis. In Proceedings of the 9th International Conference on Learning Analytics \& Knowledge (LAK19). Association for Computing Machinery, New York, NY, USA, 290-299. DOI :

http://dx.doi .org/10.1145/3303772.3303781

[7] Jeannette M Wing. 2014. Social Issues in Computing. (Jan. 2014). http:

//socialissues. cs. toronto. edu/index $\cdot h t m l ? p=279 . h t m l$

[8] Mo Zhou, Alison Cliff, Allen Huang, Sanjay Krishnan, Brandie Nonnecke, Kanji Uchino, Sam Joseph, Armando Fox, and Ken Goldberg. 2015. M-CAFE: Managing MOOC Student Feedback with Collaborative Filtering. Proceedings of the Second (2015) ACM Conference on Learning @ Scale - L@S 15 (March 2015). DOI : http://dx.doi.org/10.1145/2724660.2728681 